\title{
Students' experiences with the use of a social annotation tool to improve learning in flipped classrooms
}

\section{C.J.M. Suhre' ${ }^{1}$, J.C. Winnips ${ }^{2}$, V. de Boer ${ }^{3}$, P. Valdivia ${ }^{4}$, H.J.A. Beldhuis ${ }^{3}$}

${ }^{1}$ Department of Teacher Education, Faculty of Behavioural and Social Sciences, University of Groningen, The Netherlands, ${ }^{2}$ Department of Education and Communication Services, Faculty of Economics and Business Adminsitration, University of Groningen, The Netherlands, ${ }^{3}$ Department of Educational Innovation, Center for Information Technology, University of Groningen, The Netherlands, ${ }^{4}$ Department of European Culture \& Literature, Faculty of Arts, University of Groningen, The Netherlands.

\begin{abstract}
To support the development and dissemination of more activating educational practices, pilot studies were launched on the use of the social annotation tool Perusall. Teachers of eight higher education course units agreed to work with Perusall in their classes. To assess the usefulness and effectiveness of Perusall we focused on two related aspects (1) perception by students of Perusall as an appropriate tool to support the processing of the study texts and (2) the impact of student engagement in Perusall on students' examination results. Student experiences were evaluated by means of online questionnaires about several aspects concerning the use of Perusall and log data about student activities in the Perusall platform.

The results of the study show that Perusall can foster engagement in deep level processing of course content but that the level of processing depends on three critical factors: the transparency of annotation assignments, the perceived ease of working in Perusall and teachers' use of students' annotations to discuss the course content during lectures. The study further shows that students receive better examination results the more they engage in the annotation assignments.
\end{abstract}

Keywords: Flipped classroom; Collaboration; Academic performance. 


\section{Introduction}

In order to prepare students for a career after their studies, universities need to offer students opportunities to develop professional competences and skills as critical and creative thinking. Students in turn need to learn to seize the available opportunities to engage in critical and creative thinking and to apply what they have learned. One way to support the development of students in a role as active learners could be by designing lectures according to a flipped classroom approach, since this approach allows teachers to focus students' minds on further exploration of topics they have read before they come to class. The flipped classroom approach thus may be used to evoke discussions about learned material and to have students think about solving problems. The use of a social annotation (SA) tool could fit in with the flipped classroom concept.

In this paper we review the results of a university wide innovation pilot in which the SA tool Perusall was used by teachers of eight courses. This paper aims to investigate two related aspects of the use of Perusall in these participating course units: (1) students' perceptions of Perusall as an appropriate tool to support their processing of course content and (2) the impact of student engagement in Perusall (based on indicators derived from log data about students' activity in Perusall) on students' performance on examinations. Information about the predictive validity of engagement indicators based on log data could be useful information for teachers as it might allow them to provide early warning signals to students to increase their engagement. In this study setting we seek to address two main research questions:

1. Which aspects of the use of a social annotation tool in courses affect students' perception of engagement in deep learning?

2. Is the amount of student engagement in a social annotation tool related to the quality of their performance on assignments during the course and their examination performance?

\subsection{Implementation of Perusall and evaluation of effects of student engagement}

Perusall is an SA tool that allows teachers to ask students to study and annotate texts collaboratively before they come to class. In Perusal students create threads by highlighting text passages and posting questions or comments. Figure 1 shows the user interface of this annotation tool. 


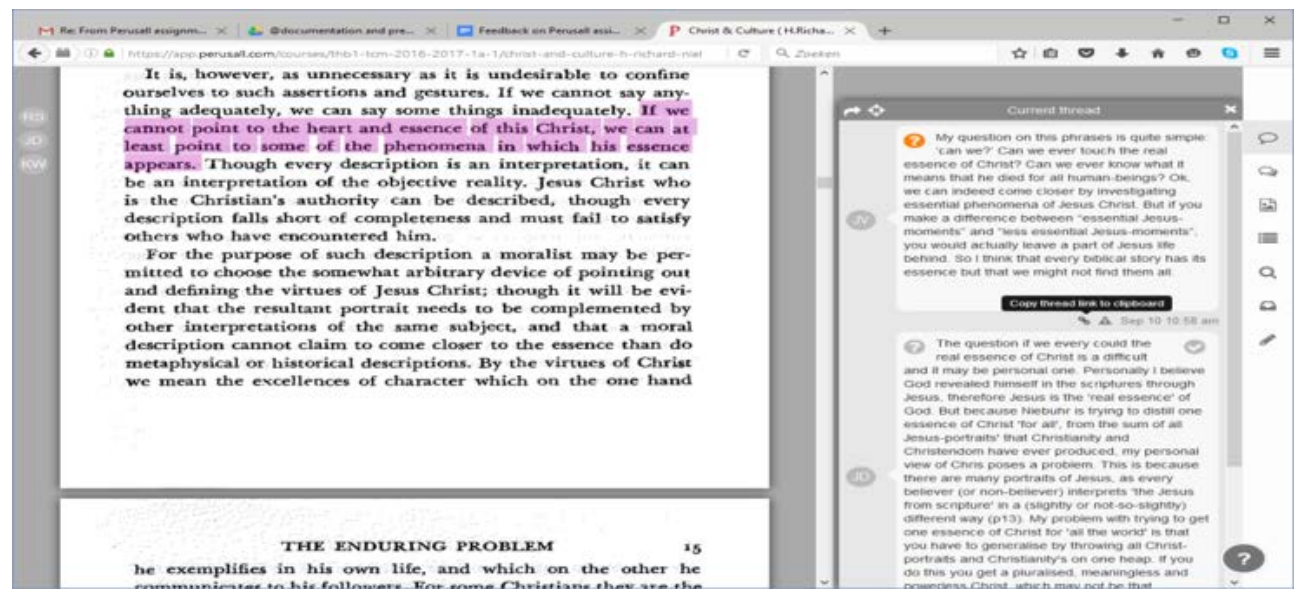

Figure 1. Example of a text fragment, as displayed in the webbrowser, with annotations and threads.

Perusall has the capacity to automatically assign scores to students' annotations as an indicator of the quality of each student's involvement in collective reading assignments. For in-depth evaluation of students' annotations to specified texts, teachers can command the Perusall platform to create so-called confusion reports that provides an overview of the content of students' annotations. By reading and analysing these reports, teacher can gain more insight into specific content that needs further clarification during lectures. This feature of Perusall can provide an impetus for both the quality of education and the professional development of teachers.

\subsection{Evaluation of student learning engagement in Perusall}

Essential for students' learning and the development of students' capacity to apply their knowledge is to ensure that students engage in reading and critical examination of texts, preferably before they come to class. This requires the use of higher order thinking skills such as comparing ideas, applying new concepts and evaluating arguments. Furthermore, those who come to class prepared are more likely to engage in discussions (Rahman \& Manaf, 2017). Student learning engagement depends both on the time students invest in studying course content as well on the tactics they apply in educationally purposeful activities (Kuh, 2002). Research by Miller et al. (2018) indicates that having students perform regular annotation assignments in a secondary science classroom does accomplish better student learning outcomes. In line with this study we focus on investigating the impact of the time students spend reading texts in Perusall during a course, the number and the quality of annotation assignments the teachers gives students to perform. 


\subsection{Exploration of factors affecting students' engagement and learning}

In this research project we studied the role of three critical factors that can facilitate or undermine student learning. It is to be expected that the quality of learning depends on:

1. the perceived ease of use of the annotation tool,

2. the nature of annotation assignments, and

3. the coverage of annotations by teachers to support students' understanding of the course content and the preparation for exams.

The ease of use pertains to reading and working within a digital environment. Transparency about the number and nature of assignments to annotate texts is a factor that could affect both students' engagement in Perusall as well as the quality of executed assignments. The perceived coverage by teachers during lectures of the information reflected in students' annotations can be an additional critical factor. Some students may need more clarification of course content, while other students value exploration and discussion of more difficult topics.

The basic framework for our investigation is displayed in Figure 2.

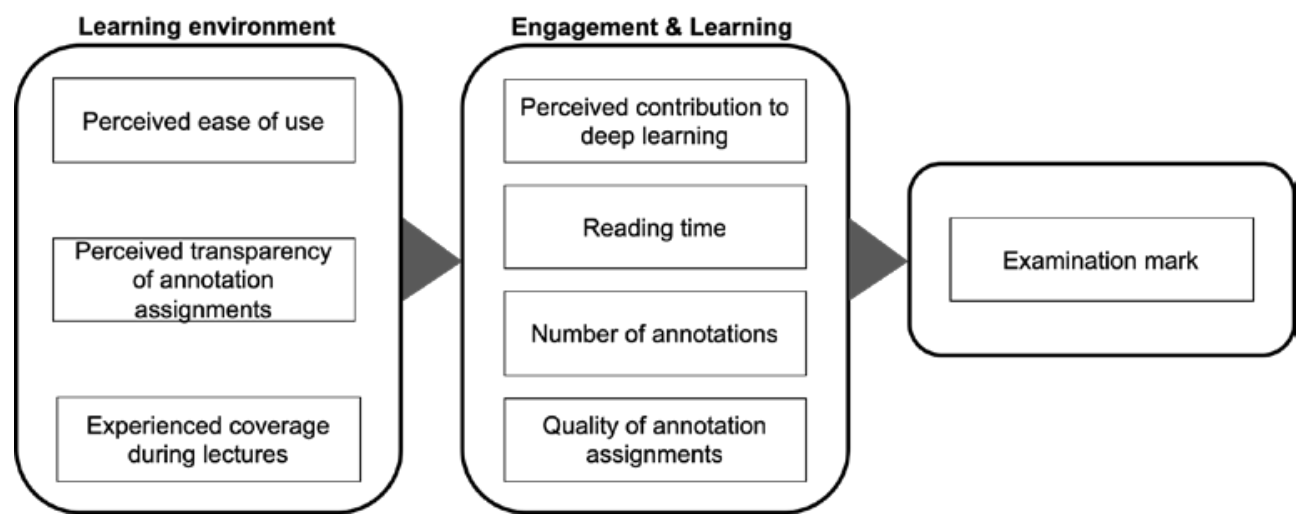

Figure 2. Framework for this study.

\section{Method}

The focus in this paper is twofold. Firstly, we studied which aspects of the use of Perusall in university courses contribute to students' perception of deep learning. We used a survey to collect data on student perceptions of the use of Perusall in their course. Secondly, we evaluated the impact of students' engagement in annotation assignments in Perusall (based on indicators derived from log data in Perusall) on students' examination performance. 


\subsection{Sample}

This study is based on data from eight courses in which Perusall was used. In these courses teachers instructed students to place 5-7 annotations per reading assignment. For these eight courses we collected data among students about their experiences with the use of Perusall and their perception of its support to learning. For these same eight courses log data are available concerning the time students spent reading documents in Perusall, the number of annotations they put in Perusall and the grading of annotations in connection to the assignments.

\subsection{Instruments}

A structured questionnaire was used to collect data about students' experiences with the use of Perusall during the course. Students could give their opinion concerning several statements with four answer options ranging from completely disagree' to 'completely agree'. The statements were categorized in four categories (perceived ease of use, transparency of annotations, coverage of annotations and contribution of working in Perusall to deep learning) that form reliable scales. Mean item scores were computed for all four scales. The reliability of the scales varied between alpha .68 and .78. To allow students to elaborate on their experience with the use of Perusall the questionnaire contained an 'additional comments' open question at the end.

In this study we further used data about students' examination marks (which range between 1 and 10, 10 indicating the maximum mark), students' overall level of examination performance in their degree programme (indicated by their average mark on course units) and log data about the time spent reading documents associated with the annotation assignments (measured by computing the time during which students scrolled, annotated or clicked within a 2 minute time interval), the number of annotations and the assignments scores. These data were aggregated to allow for subsequent multilevel regression analyses. We computed z-scores on student's average examination marks and two engagement variables: the total reading time (expressed in units of hours) and the number of annotations students put in Perusall. As an indicator of the quality of students' annotation assignments we computed the percentage of the total number of points students could achieve after completion of all assignments.

\subsection{Data collection and data preparation}

Teachers of the courses received a request to participate in research about students'perceptions of the use of Perusall in their course. In six of the eight course units the participating teachers sent their students a link to an online questionnaire. In two courses teachers requested a paper questionnaire to hand to their students. Log data about student engagement were retrieved from the servers. The log data were used to compute 
aggregate scores of student engagement. We computed students' total reading time, the number of annotations students they had put in Perusall and the percentage of the total score they could obtain by completing all annotation assignments.

\subsection{Analysis}

We assessed the impact of critical factors in the teaching setting and student engagement on deep learning and examination performance by means of multilevel analyses in which different random coefficients models were compared.

\section{Findings}

\subsection{Student perceptions about critical factors to learning in Perusall}

The descriptive survey data show that in most course units students were reasonably satisfied about the transparency of the annotation assignments but that only in two course units students' scale scores on the perceived ease of use scale indicate high degrees of satisfaction. In three of the eight course units students indicated the annotations were discussed thoroughly during lectures.

Table 1. Regression coefficients for the fixed effects of the factors transparency, ease of use and coverage of annotations in the course units on learning

\begin{tabular}{cccc}
\hline Regression coefficients & Estimate & $\mathbf{t}$ & Sig. \\
\hline Intercept & 2.65 & 44.149 & .00 \\
Transparency of annotations & .21 & 2.92 & .01 \\
Ease of use of Perusall & .46 & 6.57 & .00 \\
Coverage in lectures & .21 & 3.56 & .00 \\
\hline
\end{tabular}

By means of a multilevel analysis we evaluated the relevance of the three critical factors for students perception of the contribution of Perusall to deep learning. As can be seen in table 1, all three factors have a significant impact on the perceived contribution of the annotation tool to students' learning. The amount of variance explained is $37 \%$. Students' comments to the open question about their experiences with the use of Perusall revealed that many students had been unhappy with reading from a computer screen and expressed disconcern about the obligatory number of annotations. Especially students who started late reading the text before class expressed difficulty posting meaningful questions or answers to questions of other students. 


\subsection{Impact of student engagement on the final examination marks}

In a second series of analyses we evaluated the relationship between student engagement in Perusall and both students' performance on the annotations and the final mark of the concluding examination. The first analysis revealed that differences in students' scores on the annotation assignments depend on both the total reading time and the total number of annotations, the total number of annotations having the largest impact. A further analysis of the impact of successful engagement in the annotation assignments on students' examination performance indicates that there is a positive relationship between the scores obtained on the annotation assignments during the course and the final examination mark. The results of this analysis are displayed in Table 2.

Table 2. Regression coefficients for the fixed effects of the factors assignments score and average examination mark

\begin{tabular}{cccc}
\hline Regression coefficients & Estimate & $\mathbf{t}$ & Sig. \\
\hline Intercept & 5.79 & 13.59 & .00 \\
Assignments score (\%) & .02 & 5.93 & .00 \\
Average examination mark & .62 & 4.76 & .00 \\
\hline
\end{tabular}

The regression coefficients in table 2 indicate that students' examination marks depend both on students' annotation assignments scores and (to a very high extent) on their average level of performance in other courses (average mark). In all courses in which Perusall was used the difference in examination mark between a student with the same average level of performance who has a score of $50 \%$ and one who has a $100 \%$ score on the assignments is approximately 1 point on a 1-10 grading scale. The size of the impact of the obtained assignment scores on students' examination marks is about the same in all courses as evidenced by the absence of a significant difference in chi-square between a multilevel model with and without a random regression coefficient. This multilevel model explains 22 percent of the variance of the examination grades.

\section{Discussion}

The results of this study indicate that the SA tool Perusall contributes to deep learning and that students who perform the annotation assignments as required obtain significantly higher examination marks. Perusall can thus be considered an effective learning tool for students. However, despite the positive outcomes of the pilots, his study also reveals that the contribution to students depth of learning depends on their perception of ease of use, transparency of annotation assignments and teachers' coverage of annotations. Students' complaints about the ease of use not only concern technical features of the tool such as 
awkwardness of reading from the screen, printing or downloading documents but also practical issues such as having difficulty in adding meaningful annotations when other students had already added annotations to a text. In some course units students judged the transparency of assessment of their annotation tasks and the discussion of annotations in class by their teachers insufficient.

Given the expressed concerns above, teachers should carefully consider the choice of text documents, the nature of annotation assignments and the relevance of these assignments for students' preparation on final examinations. Teachers should take care that:

- $\quad$ selected texts and the group size allow for sufficient meaningful annotations.

- the nature of the assignments stimulate students to reply to each other's annotations.

- grading of annotations is clear and students receive examples of good annotations.

- $\quad$ students receive timely feedback on the content of their annotations.

\section{References}

Davis, F. D. (1989). Perceived usefulness, perceived ease of use, and user acceptance of information technology. MIS Quarterly, 13(3), 319-340.

Kuh, G. D. (2002). The National Survey of Student Engagement: Conceptual framework and overview of psychometric properties. Available at: http://nsse.indiana.edu/pdf/psychometric_framework_2002.pdf

Miller, K., Lukoff, B., King, G., \& Mazur, E. (2018). Use of a social annotation platform for pre-class reading assignments in a flipped introductory physics class. Frontiers in Education, 3, 1-12.

Rahman, S. A., \& Manaf, N. F. A. (2017). A critical analysis of Bloom's taxonomy in teaching creative and critical thinking skills in Malaysia through English literature. English Language Teaching, 10(9), 245-256. 